\title{
Scattering theory with path integrals
}

\author{
R. Rosenfelder \\ Particle Theory Group, Paul Scherrer Institute, CH-5232 Villigen PSI, Switzerland
}

(Received 27 June 2013; accepted 15 February 2014; published online 13 March 2014)

Starting from well-known expressions for the $T$-matrix and its derivative in standard nonrelativistic potential scattering, I rederive recent path-integral formulations due to Efimov and Barbashov et al. Some new relations follow immediately. ㄷ 2014 AIP

Publishing LLC. [http://dx.doi.org/10.1063/1.4867605]

\section{INTRODUCTION}

Traditionally, the path-integral method in quantum physics has been applied mostly to boundstate problems. Following the pioneering work of Ref. 1, there has been renewed interest in the last few years to use it also for scattering problems ${ }^{2-4}$ in nonrelativistic physics. This offers the chance of finding new approximation methods ${ }^{6,7}$ or trying to evaluate the involved path integrals by stochastic methods although the oscillating nature of the real-time path integral presents a great challenge (see, e.g., Ref. 8). It should be also kept in mind that the path-integral approach, where one integrates functionally over the degrees of freedom weighted by the exponential of the classical action, is much more general than a Schrödinger description thus allowing an immediate generalization to many-body or field-theoretical problems. ${ }^{9-11}$ In the following, however, only singlechannel nonrelativistic scattering in a local potential $V(\mathbf{x})$ is considered with the aim to find practical path-integral representations for the scattering amplitude free of infinite (time-)limits. Extensions to nonlocal potentials have been considered in Ref. 8.

Most of the results so far have been derived by non-standard methods specific for path integrals. For example, in Ref. 2 the $S$-matrix has been considered as infinite-time limit of the evolution operator in the interaction picture but the extraction of the energy-conserving $\delta$-function, i.e., an expression for the $T$-matrix was only possible by applying the Faddeev-Popov trick and introducing "anti-velocity" degrees of freedom which take away unphysical contributions in the path integral. In the meantime path-integral formulations for the $T$-matrix have been found (or rediscovered ${ }^{3}$ ) which do not need an "anti-velocity" at all but are more involved. They are either based on a technique invented by Barbashov and collaborators in quantum field theory ${ }^{12}$ or use the asymptotic limit of the path-integral solution of the Schrödinger equation. ${ }^{4,5}$

One may wonder whether it is not possible to start from well-developed nonrelativistic scattering theory as available in many excellent text books. ${ }^{13-17}$ This is the aim of the present note and, indeed, I succeed in deriving old - and also some new - path-integral expressions for the scattering amplitude which may be of some use for further analytical or numerical studies. To be self-contained, some additional material about velocity path integrals and derivatives of the $T$-matrix is collected in Appendices A and B.

\section{FINAL (EFIMOV'S) FORM FOR THE T-MATRIX}

Here we start from the standard expression ${ }^{18}$ for the $T$-matrix for scattering from a local potential $V(\mathbf{x})$ (see, e.g., Ref. 13, Eq. (XIX.17))

$$
T_{i \rightarrow f}=\left\langle\psi_{f}^{[-)}|\hat{V}| \phi_{i}\right\rangle
$$


where $\left\langle\mathbf{x} \mid \phi_{i}\right\rangle=\exp \left(i \mathbf{k}_{i} \cdot \mathbf{x}\right)$ is a free plane wave with initial momentum $\mathbf{k}_{i}$ and

$$
\left\langle\psi_{f}^{(-)}\right|=\left\langle\phi_{f}\right|\left(\hat{U}_{I}(0,+\infty)\right)^{\dagger}=\left\langle\phi_{f}\right| \hat{U}_{I}(+\infty, 0)
$$

is the full incoming scattering wave (Ref. 14, Chap. 8, Eq. (3.4)) with final momentum $\mathbf{k}_{f}$. The latter is obtained from the free wave by application of the Møller operator

$$
\hat{U}_{I}(+\infty, 0)=\left.\lim _{t_{1} \rightarrow \infty} e^{i \hat{H}_{0} t_{1}} \hat{U}\left(t_{1}, t_{0}\right) e^{-i \hat{H}_{0} t_{0}}\right|_{t_{0}=0},
$$

which is the time-evolution operator in the interaction picture associated with the free/full Hamiltonians $\hat{H}_{0} / \hat{H}$ (Ref. 14, Chap. 8, Eq. (1.22)). The delicate infinite-time limit can be controlled by making the potential slightly time-dependent, e.g., by multiplying it with a factor $\exp (-\epsilon|t|)$ and setting $\epsilon=0$ at the very end of the calculation, i.e., by switching off the interaction at very early and very late times. If we insert Eq. (3) into Eq. (1) we obtain

$$
\begin{aligned}
T_{i \rightarrow f} & =\lim _{T \rightarrow \infty} e^{i E T}\left\langle\phi_{f}|\hat{U}(T, 0) \hat{V}| \phi_{i}\right\rangle \\
& =\lim _{T \rightarrow \infty} e^{i E T} \int d^{3} x d^{3} y\left\langle\phi_{f} \mid \mathbf{y}\right\rangle\langle\mathbf{y}|\hat{U}(T, 0)| \mathbf{x}\rangle V(\mathbf{x})\left\langle\mathbf{x} \mid \phi_{i}\right\rangle,
\end{aligned}
$$

where $E=\mathbf{k}_{i}^{2} /(2 m)=\mathbf{k}_{f}^{2} /(2 m)$ is the scattering energy, and we now can employ the path-integral representation (A8) for the full time-evolution operator as presented in Appendix A. This gives

$$
\begin{aligned}
T_{i \rightarrow f}= & \lim _{T \rightarrow \infty} e^{i E T} \int d^{3} x d^{3} y e^{-i \mathbf{k}_{f} \cdot \mathbf{y}}\left(\frac{m}{2 \pi i T}\right)^{3 / 2} \exp \left[i \frac{m}{2} \frac{(\mathbf{y}-\mathbf{x})^{2}}{T}\right] \\
& \times \mathcal{N}_{\xi} \int_{\boldsymbol{\xi}_{(0)=0}}^{\boldsymbol{\xi}(T)=0} \mathcal{D}^{3} \xi \exp \left\{i \int_{0}^{T} d t\left[\frac{m}{2} \dot{\xi}^{2}-V\left(\mathbf{x}+\frac{\mathbf{y}-\mathbf{x}}{T} t+\boldsymbol{\xi}(t)\right)\right]\right\} V(\mathbf{x}) e^{i \mathbf{k}_{i} \cdot \mathbf{x}} .
\end{aligned}
$$

The normalization $\mathcal{N}_{\xi}$ (Eq. (A9) with $t_{b}-t_{a}=T$ ) ensures that the free path integral gives unity. After substituting $\mathbf{y}=\mathbf{x}+\mathbf{u} T$ we can perform the $\mathbf{u}$-integration in the large $T$-limit by the stationary phase method

$$
\left(\frac{m T}{2 \pi i}\right)^{3 / 2} \int d^{3} u \exp \left[i\left(-\mathbf{k} \cdot \mathbf{u}+\frac{m}{2} \mathbf{u}^{2}\right) T\right] F(\mathbf{u}) \stackrel{T \rightarrow \infty}{\simeq} \exp \left(-i \frac{\mathbf{k}^{2}}{2 m} T\right) F\left(\mathbf{u}_{\text {stat }}\right)
$$

with the stationary point

$$
\mathbf{u}_{\text {stat }}=\frac{\mathbf{k}}{m}, \quad \mathbf{k} \equiv \mathbf{k}_{f}
$$

if the function $F(\mathbf{u})$ does not vary much. Therefore, all explicit $T$-dependence completely cancels out and the final result is

$T_{i \rightarrow f}=\int d^{3} x e^{-i \mathbf{q} \cdot \mathbf{x}} V(\mathbf{x}) \mathcal{N}_{\xi} \int_{\boldsymbol{\xi}(0)=0}^{\boldsymbol{\xi}(\infty)=0} \mathcal{D}^{3} \xi \exp \left\{i \int_{0}^{\infty} d t\left[\frac{m}{2} \dot{\xi}^{2}-V\left(\mathbf{x}+\frac{\mathbf{k}_{f}}{m} t+\boldsymbol{\xi}(t)\right)\right]\right\}$,

where $\mathbf{q}=\mathbf{k}_{f}-\mathbf{k}_{i}$ denotes the momentum transfer. This obviously reduces to the first Born approximation if the path integral, i.e., higher orders, are neglected. It is also in agreement with the result given by Efimov ${ }^{4}$ for the scattering amplitude $f_{i \rightarrow f}=-m T_{i \rightarrow f} /(2 \pi) .^{19}$

\section{INITIAL FORM FOR THE T-MATRIX}

There is an alternative form of the $T$-matrix in scattering theory (given, e.g., in Ref. 13, Eq. (XIX.15))

$$
T_{i \rightarrow f}=\left\langle\phi_{f}|\hat{V}| \psi_{i}^{(+)}\right\rangle
$$

where the full outgoing scattering wave function with momentum $\mathbf{k}_{i}$

$$
\left|\psi_{i}^{(+)}\right\rangle=\hat{U}_{I}(0,-\infty)\left|\phi_{i}\right\rangle
$$


enters (Ref. 14, Chap. 8, Eq. (3.2)). Proceeding as in Sec. II we obtain

$$
\begin{aligned}
T_{i \rightarrow f}= & \lim _{T \rightarrow \infty} e^{i E T} \int d^{3} x d^{3} y e^{-i \mathbf{k}_{f} \cdot \mathbf{x}} V(\mathbf{x})\left(\frac{m}{2 \pi i T}\right)^{3 / 2} \exp \left[i \frac{m}{2} \frac{(\mathbf{x}-\mathbf{y})^{2}}{T}\right] \\
& \times \mathcal{N}_{\xi} \int_{\boldsymbol{\xi}_{(-T)=0}}^{\boldsymbol{\xi}(0)=0} \mathcal{D}^{3} \xi \exp \left\{i \int_{-T}^{0} d t\left[\frac{m}{2} \dot{\boldsymbol{\xi}}^{2}-V\left(\mathbf{y}+\frac{\mathbf{x}-\mathbf{y}}{T}(t+T)+\boldsymbol{\xi}(t)\right)\right]\right\} e^{i \mathbf{k}_{i} \cdot \mathbf{y}} .
\end{aligned}
$$

Again we perform the substitution $\mathbf{y}=\mathbf{x}-\mathbf{u} T$, find the stationary point in the $\mathbf{u}$-integral as $\mathbf{u}_{\text {stat }}$ $=\mathbf{k}_{i} / m$, and obtain

$$
T_{i \rightarrow f}=\int d^{3} x e^{-i \mathbf{q} \cdot \mathbf{x}} V(\mathbf{x}) \mathcal{N}_{\xi} \int_{\boldsymbol{\xi}_{(-\infty)=0}}^{\boldsymbol{\xi}(0)=0} \mathcal{D}^{3} \xi \exp \left\{i \int_{-\infty}^{0} d t\left[\frac{m}{2} \dot{\boldsymbol{\xi}}^{2}-V\left(\mathbf{x}+\frac{\mathbf{k}_{i}}{m} t+\boldsymbol{\xi}(t)\right)\right]\right\}
$$

It is seen that the transformations $\mathbf{k}_{i} \rightarrow-\mathbf{k}_{f}, \mathbf{k}_{f} \rightarrow-\mathbf{k}_{i}$ together with the change $t \rightarrow-t$ in the $t$-integral turn Eq. (12) into Eq. (8). Thus, our path-integral representations fulfill the property of microreversibility (Ref. 13, Eq. (XIX.21)) or time-reversal invariance

$$
T_{(-f) \rightarrow(-i)}=T_{i \rightarrow f} .
$$

\section{SYMMETRIC (BARBASHOV'S) FORM FOR THE T-MATRIX}

The path-integral expressions (12) and (8) are both exact but emphasize the initial and final momentum, respectively, as do the starting points (9) and (1). A more symmetrical form would be useful, in particular when approximations are made. Otherwise time-reversal invariance could be violated. Of course, the arithmetic average of both expressions would suffice but this is a rather awkward procedure. What is needed is an expression for the $T$-matrix in which both ingoing and outgoing scattering waves are contained.

This is provided by an expression for the derivative of the $T$-matrix with respect to a parameter $\lambda$ of the potential ${ }^{20}$

$$
\frac{\partial T_{i \rightarrow f}}{\partial \lambda}=\left\langle\psi_{f}^{(-)}\left|\frac{\partial \hat{V}_{\lambda}}{\partial \lambda}\right| \psi_{i}^{(+)}\right\rangle
$$

a proof of which is given in Appendix B. Writing

$$
\begin{aligned}
\frac{\partial T_{i \rightarrow f}}{\partial \lambda} & =\left\langle\phi_{f}\left|\hat{U}_{I}(\infty, 0) \frac{\partial \hat{V}_{\lambda}}{\partial \lambda} \hat{U}_{I}(0,-\infty)\right| \phi_{i}\right\rangle \\
& =\lim _{T \rightarrow \infty} e^{2 i E T}\left\langle\phi_{f}\left|\hat{U}(T, 0) \frac{\partial \hat{V}_{\lambda}}{\partial \lambda} \hat{U}(0,-T)\right| \phi_{i}\right\rangle
\end{aligned}
$$

we now insert the path integral representation (A12) for matrix elements of the time-evolution operators and obtain

$$
\begin{aligned}
\frac{\partial T_{i \rightarrow f}}{\partial \lambda} & =\lim _{T \rightarrow \infty} e^{2 i E T} \int d^{3} x_{0} d^{3} x_{1} d^{3} x_{2} e^{-i \mathbf{k}_{f} \cdot \mathbf{x}_{2}} \frac{\partial V_{\lambda}\left(\mathbf{x}_{1}\right)}{\partial \lambda} e^{i \mathbf{k}_{i} \cdot \mathbf{x}_{0}} \\
& \times \prod_{k=1}^{2} \mathcal{N}_{v_{k}} \int \mathcal{D}^{3} v_{k} \delta^{(3)}\left(\mathbf{x}_{k}-\mathbf{x}_{k-1}-\int_{T_{k-1}}^{T_{k}} d t_{k} \mathbf{v}_{k}\right) \exp \left\{i \int_{T_{k-1}}^{T_{k}} d \tau_{k}\left[\frac{m}{2} \mathbf{v}_{k}^{2}-V\left(\mathbf{y}_{k}\right)\right]\right\}
\end{aligned}
$$

Here we have used functional integration over velocities $\mathbf{v}_{k} \equiv \mathbf{v}_{k}\left(t_{k}\right), k=1,2$ which has the advantage that no boundary conditions have to be observed (see Appendix A). The normalizations 
$\mathcal{N}_{v_{k}}$ are again such that the free path integrals are one (see Eq. (A14)). The arguments of the potential terms read (cf. Eq. (A13))

$$
\mathbf{y}_{k}\left(\tau_{k}\right)=\frac{\mathbf{x}_{k}+\mathbf{x}_{k-1}}{2}+\mathbf{x}_{v_{k}}\left(\tau_{k}\right), \quad k=1,2,
$$

where

$$
\mathbf{x}_{v_{k}}\left(\tau_{k}\right):=\frac{1}{2}\left[\int_{T_{k-1}}^{\tau_{k}} d s \mathbf{v}_{k}(s)-\int_{\tau_{k}}^{T_{k}} d s \mathbf{v}_{k}(s)\right] \quad \text { with } \quad T_{0}:=-T, T_{k}=T_{k-1}+T
$$

are the quantum fluctuations around the mean position. Eliminating the coordinates $\mathbf{x}_{0}, \mathbf{x}_{2}$ by means of the two $\delta$-functions one obtains

$$
\mathbf{y}_{k}\left(\tau_{k}\right)=\mathbf{x}_{1}+\int_{0}^{\tau_{k}} d s \mathbf{v}_{k}(s), \quad \tau_{k} \in\left[T_{k-1}, T_{k}\right]
$$

and

$$
-\mathbf{k}_{f} \cdot \mathbf{x}_{2}+\mathbf{k}_{i} \cdot \mathbf{x}_{0}=-\mathbf{q} \cdot \mathbf{x}_{1}-\mathbf{k}_{i} \cdot \int_{-T}^{0} d t_{1} \mathbf{v}_{1}-\mathbf{k}_{f} \cdot \int_{0}^{T} d t_{2} \mathbf{v}_{2} .
$$

If we now define

$$
\mathbf{v}(t):= \begin{cases}\mathbf{v}_{1}(t)+\mathbf{k}_{i} / m & t \in[-T, 0] \\ \mathbf{v}_{2}(t)+\mathbf{k}_{f} / m & t \in[0,+T]\end{cases}
$$

and extend the time interval from $-T$ to $+T$, we can concentrate the functional integration into one velocity variable $\mathbf{v}(t)$ as in the proof of the composition law of the time-evolution operator in Appendix A. Writing $\mathbf{x}$ for $\mathbf{x}_{1}$ we then have

$$
\begin{aligned}
\frac{\partial T_{i \rightarrow f}}{\partial \lambda}= & \int d^{3} x e^{-i \mathbf{q} \cdot \mathbf{x}} \frac{\partial V_{\lambda}(\mathbf{x})}{\partial \lambda} \mathcal{N}_{v} \\
& \times \int \mathcal{D}^{3} v \exp \left\{i \int_{-\infty}^{+\infty} d t \frac{m}{2} \mathbf{v}^{2}(t)-i \int_{-\infty}^{+\infty} d t V\left(\mathbf{x}+\boldsymbol{\rho}_{\text {ray }}(t, \mathbf{v}]\right)\right\},
\end{aligned}
$$

where $^{21}$

$$
\boldsymbol{\rho}_{\text {ray }}(t, \mathbf{v}]=\left[\frac{\mathbf{k}_{i}}{m} \Theta(-t)+\frac{\mathbf{k}_{f}}{m} \Theta(t)\right] t+\int_{0}^{t} d \tau \mathbf{v}(\tau)=: \mathbf{x}_{\text {ray }}(t)+\mathbf{x}_{\text {quant }}(t, \mathbf{v}] .
$$

Note that again all dependence on the large time $T$ has disappeared because $\mathbf{k}_{i}^{2} /(2 m)+\mathbf{k}_{f}^{2} /(2 m)$ $=2 E$. It is seen that the particle mainly travels along the ray formed by the initial and final momentum while the functional integration supplies the quantum fluctuations around that path.

As a special case of Eqs. (14) and (22) we multiply the original potential by a strength parameter $\lambda \in[0,1]$. Integrating over $\lambda$ from zero to one ${ }^{22}$ we immediately obtain from Eq. (22) the result of Ref. 3

$$
\begin{aligned}
T_{i \rightarrow f}= & \int d^{3} x e^{-i \mathbf{q} \cdot \mathbf{x}} V(\mathbf{x}) \mathcal{N}_{v} \int \mathcal{D}^{3} v \exp \left[i \int_{-\infty}^{+\infty} d t \frac{m}{2} \mathbf{v}^{2}(t)\right] \int_{0}^{1} d \lambda \\
& \times \exp \left[-i \lambda \int_{-\infty}^{+\infty} d t V\left(\mathbf{x}+\boldsymbol{\rho}_{\text {ray }}(t, \mathbf{v}]\right)\right]
\end{aligned}
$$

which was previously derived using a technique developed by Barbashov et al. ${ }^{12}$ in quantum field theory. Here we see that this time-reversal invariant representation follows from standard scattering theory.

As outlined in Appendix A any velocity path integral can also be written as ordinary (Feynman) path integral. Taking into account the relation

$$
\mathbf{x}_{\text {quant }}(t, \mathbf{v}]=\int_{0}^{t} d \tau \mathbf{v}(\tau)=\mathbf{x}_{v}(t)-\mathbf{x}_{v}(0)
$$


and applying Eq. (A17) to Eq. (24) one obtains

$$
\begin{aligned}
T_{i \rightarrow f}= & \int d^{3} x e^{-i \mathbf{q} \cdot \mathbf{x}} V(\mathbf{x}) \lim _{T \rightarrow \infty} \int d^{3} z\left(\frac{m}{2 \pi i T}\right)^{3 / 2} \exp \left(i \frac{m \mathbf{z}^{2}}{2 T}\right) \mathcal{N}_{\xi} \int_{\boldsymbol{\xi}(-T / 2)=0}^{\boldsymbol{\xi}(+T / 2)=0} \mathcal{D}^{3} \xi \\
& \times \exp \left[i \int_{-T / 2}^{+T / 2} d t \frac{m}{2} \dot{\xi}^{2}(t)\right] \int_{0}^{1} d \lambda \exp \left[-i \lambda \int_{-T / 2}^{+T / 2} d t V\left(\mathbf{x}+\frac{\mathbf{z}}{T} t+\tilde{\boldsymbol{\rho}}_{\text {ray }}(t, \boldsymbol{\xi}]\right)\right],
\end{aligned}
$$

where

$$
\tilde{\boldsymbol{\rho}}_{\text {ray }}(t, \boldsymbol{\xi}]=\mathbf{x}_{\text {ray }}(t)+\boldsymbol{\xi}(t)-\boldsymbol{\xi}(0) \text {. }
$$

Substituting $\mathbf{z}=\mathbf{u} T$ we can again evaluate the $\mathbf{u}$-integral in the limit $T \rightarrow \infty$ by the method of stationary phase as given by Eq. (6), but now with $\mathbf{k}=0, \mathbf{u}_{\text {stat }}=0$. This leads to the new expression

$$
\begin{aligned}
T_{i \rightarrow f}= & \int d^{3} x e^{-i \mathbf{q} \cdot \mathbf{x}} V(\mathbf{x}) \mathcal{N}_{\xi} \int_{\boldsymbol{\xi}(-\infty)=0}^{\boldsymbol{\xi}_{(+\infty)}} \mathcal{D}^{3} \xi \exp \left[i \int_{-\infty}^{+\infty} d t \frac{m}{2} \dot{\boldsymbol{\xi}}^{2}(t)\right] \int_{0}^{1} d \lambda \\
& \times \exp \left[-i \lambda \int_{-\infty}^{+\infty} d t V(\mathbf{x}+\tilde{\boldsymbol{\rho}}(t, \boldsymbol{\xi}])\right] .
\end{aligned}
$$

Compared to the velocity path integral (24) this Feynman path integral for the $T$-matrix has one integration less (not a big deal for an infinite-dimensional functional integral. . .) but the paths have to obey specific boundary conditions. Therefore, the jury is still out on deciding which form is more convenient - for the analytic task of checking the second Born approximation the path integral in Eq. (28) seems to be more involved than the one in Eq. (24) which only involves Gaussian integration. For other applications, the representation (28) by an ordinary path integral may offer advantages.

Finally, I will give a path-integral expression for the derivative of the $T$-matrix with respect to the momentum $k=\left|\mathbf{k}_{i}\right|=\left|\mathbf{k}_{f}\right|$ starting from the symmetric expression ${ }^{20,23,24}$

$$
\frac{\partial}{\partial k}\left(k T_{i \rightarrow f}\right)=-\left\langle\psi_{f}^{(-)}|2 V(\hat{\mathbf{x}})+\hat{\mathbf{x}} \cdot \nabla V(\hat{\mathbf{x}})| \psi_{i}^{(+)}\right\rangle .
$$

As this relation can be derived by a similar scaling of coordinate and momentum as the virial theorem in the bound-state case (see Appendix B) one may call it the "scattering virial theorem." Following the derivation of Eq. (22) its path-integral representation can be written down immediately

$$
\begin{aligned}
\frac{\partial}{\partial k}\left(k T_{i \rightarrow f}\right)=-\int d^{3} x e^{-i \mathbf{q} \cdot \mathbf{x}} & {[2 V(\mathbf{x})+\mathbf{x} \cdot \nabla V(\mathbf{x})] \mathcal{N}_{v} \int \mathcal{D}^{3} v \exp \left[i \int_{-\infty}^{+\infty} d t \frac{m}{2} \mathbf{v}^{2}(t)\right] } \\
& \times \int_{0}^{1} d \lambda \exp \left[-i \lambda \int_{-\infty}^{+\infty} d t V\left(\mathbf{x}+\boldsymbol{\rho}_{\text {ray }}(t, \mathbf{v}]\right)\right] .
\end{aligned}
$$

A similar expression could be given for the derivative of the $T$-matrix with respect to the mass $m$ of the quantum-mechanical particle starting from Eq. (13) in Ref. 20.

\section{SUMMARY AND OUTLOOK}

I have derived old (and new) path-integral expressions for the $T$-matrix in nonrelativistic potential scattering starting from well-known representations of $T$ in standard scattering theory. In this way, a recent representation due to Efimov ${ }^{4}$ in which the final momentum is emphasized has been rederived and an alternative form has been obtained in which the particle mainly propagates along the initial momentum. Of course, if the full quantum fluctuations are taken into account both forms are equivalent. Explicit time-reversal invariance requires a formalism symmetric in initial and final 
states-available from an expression for the derivative of the $T$-matric with respect to parameters of the potential or the energy. This gave the same result as previously ${ }^{3}$ derived from the field-theoretic work of Barbashov et al. ${ }^{12}$ While this result is written in terms of velocity path integrals, I also have succeeded to give a new representation by ordinary Feynman path integrals.

It is tempting to choose the $S$-matrix itself as infinite time-limit of the time-evolution operator in the interaction representation

$$
S_{i \rightarrow f}=\lim _{T \rightarrow \infty}\left\langle\phi_{f}\left|\hat{U}_{I}(T,-T)\right| \phi_{i}\right\rangle=\lim _{T \rightarrow \infty} e^{i\left(E_{i}+E_{f}\right) T}\left\langle\phi_{f}|\hat{U}(T,-T)| \phi_{i}\right\rangle
$$

which also exhibits the explicit time-reversal symmetry and perform similar steps. However, then one has to enforce energy conservation by a Faddeev-Popov-like constraint in the path integral and - choosing the simplest form of this constraint ${ }^{2}$ - one ends up with a formulation where the particle travels along the mean momentum $\mathbf{K}=\left(\mathbf{k}_{i}+\mathbf{k}_{f}\right) / 2$ which is not on-shell since $K \equiv|\mathbf{K}|=k \cos \Theta / 2$ with $\Theta$ being the scattering angle. Therefore, the "dangerous" time-dependent phase $\exp \left(i\left(E_{i}+\right.\right.$ $\left.E_{f}\right) T$ ) is not cancelled and one has to introduce "phantom" degrees of freedom, i.e., "anti-velocities" to achieve that. The result

$$
\begin{aligned}
T_{i \rightarrow f}= & i \frac{K}{m} \int d^{2} b e^{-i \mathbf{q} \cdot \mathbf{b}} \mathcal{N}_{v} \mathcal{N}_{w} \int \mathcal{D}^{3} v \mathcal{D}^{d} w \\
& \times \exp \left\{i \int_{-\infty}^{+\infty} d t \frac{m}{2}\left[\mathbf{v}^{2}(t)-w^{2}(t)\right]\right\}\left\{e^{i \chi(\mathbf{b}, \mathbf{v}, w]}-1\right\}
\end{aligned}
$$

comes in two versions which are distinguished by the reference path along which the particle dominantly travels and the dimensionality $d$ of the "anti-velocity" $w(t)$. In the first case the reference path is a straight-line path along the mean momentum $\mathbf{K}$ and a $(d=3)$-dimensional anti-velocity is needed whereas in the second case the reference path is the same ray along the initial momentum and along the final momentum which appeared in the symmetric form (24) with a $(d=1)$-dimensional anti-velocity parallel to the mean momentum K. For more details see Ref. 2.

At first sight Eq. (31) looks like an impact-parameter representation of the $T$-matrix since one integrates over the 2-dimensional vector $\mathbf{b}$ which is perpendicular to the mean momentum $\mathbf{K}$, but it is not in a strict sense. This is because of the dependence of the phase $\chi(\mathbf{b}, \mathbf{v}, w]$ on the kinematic variables and the angle-dependent factor $K=k \cos (\Theta / 2)$ in front of the impact-parameter integral: In a genuine impact-parameter representation all dependence on the scattering angle $\Theta$ should only reside in the factor $\exp (-i \mathbf{q} \cdot \mathbf{b}){ }^{25}$ Although the (initial, final, or symmetric) path-integral formulations derived in this note may be quite useful in many applications they are also not in the form of an impact parameter representation. This is obvious since Eqs. (12), (8) or (24), (28) come all as a "level 1"-representation 3 of the scattering amplitude where one power of the potential appears in front of the path integral whereas an impact-parameter representation would belong to the "level 0 '"-class.

It remains an open problem to find a - practical not only formal - path-integral expression for the impact-parameter representation of the scattering amplitude.

\section{ACKNOWLEDGMENTS}

I would like to thank G. Efimov for useful correspondence.

\section{APPENDIX A: ORDINARY AND VELOCITY PATH INTEGRALS}

Consider a quantum-mechanical particle of mass $m$ in $d$ (euclidean) dimensions under the influence of a general (even time-dependent) potential $V(x, t)$.

The standard path integral expression for the matrix element of the time evolution operator ${ }^{9}$

$$
U\left(x_{b}, t_{b} ; x_{a}, t_{a}\right) \equiv\left\langle x_{b}\left|\hat{U}\left(t_{b}, t_{a}\right)\right| x_{a}\right\rangle=\left\langle x_{b}\left|\mathcal{T} \exp \left[-i \int_{t_{a}}^{t_{b}} d \tau\left(\frac{\hat{p}^{2}}{2 m}+V(\hat{x}, \tau)\right)\right]\right| x_{a}\right\rangle
$$


is obtained by slicing the time interval in $N$ pieces of size $\epsilon=\left(t_{b}-t_{a}\right) / N$, decomposing

$$
\hat{U}\left(t_{b}, t_{a}\right)=\prod_{k=1}^{N} \hat{U}\left(t_{k}, t_{k-1}\right), \quad t_{k}=t_{a}+k \epsilon
$$

using a short-time approximation for the individual factors and performing the limit $N \rightarrow \infty$ while keeping the time difference $t_{b}-t_{a}$ fixed. This gives

$$
\begin{aligned}
U\left(x_{b}, t_{b} ; x_{a}, t_{a}\right)= & \lim _{N \rightarrow \infty}\left(\frac{m}{2 \pi i \epsilon}\right)^{d N / 2} \int_{-\infty}^{+\infty} d^{d} x_{1} d^{d} x_{2} \ldots d^{d} x_{N-1} \\
& \times \exp \left\{i \epsilon \sum_{k=1}^{N}\left[\frac{m}{2}\left(\frac{x_{k}-x_{k-1}}{\epsilon}\right)^{2}-V\left(x_{k}, t_{k}\right)\right]\right\}, x_{0} \equiv x_{a}, x_{N} \equiv x_{b} \\
\equiv & \int_{x\left(t_{a}\right)=x_{a}}^{x\left(t_{b}\right)=x_{b}} \mathcal{D}^{d} x(t) \exp \left\{i \int_{t_{a}}^{t_{b}} d t\left[\frac{m}{2} \dot{x}^{2}(t)-V(x(t), t)\right]\right\}
\end{aligned}
$$

Note that no time-ordering operator $\mathcal{T}$ is needed in the path-integral formulation since it works with ordinary numbers. To get rid of the cumbersome prefactors in the discretized form we normalize all path integrals to the corresponding path integral without interaction, i.e., we use

$$
\int_{x\left(t_{a}\right)=x_{a}}^{x\left(t_{b}\right)=x_{b}} \mathcal{D}^{d} x(t) \exp \left\{i \int_{t_{a}}^{t_{b}} d t \frac{m}{2} \dot{x}^{2}(t)\right\}=\left[\frac{m}{2 \pi i\left(t_{b}-t_{a}\right)}\right]^{d / 2} \exp \left[i \frac{m}{2} \frac{\left(x_{b}-x_{a}\right)^{2}}{t_{b}-t_{a}}\right]=: \mathcal{N}_{x}^{-1} .
$$

Then

$$
\begin{aligned}
U\left(x_{b}, t_{b} ; x_{a}, t_{a}\right)= & {\left[\frac{m}{2 \pi i\left(t_{b}-t_{a}\right)}\right]^{d / 2} \exp \left[i \frac{m}{2} \frac{\left(x_{b}-x_{a}\right)^{2}}{t_{b}-t_{a}}\right] } \\
& \times \mathcal{N}_{x} \int_{x\left(t_{a}\right)=x_{a}}^{x\left(t_{b}\right)=x_{b}} \mathcal{D}^{d} x(t) \exp \left\{i \int_{t_{a}}^{t_{b}} d t\left[\frac{m}{2} \dot{x}^{2}(t)-V(x(t), t)\right]\right\} .
\end{aligned}
$$

Often it is convenient to introduce a reference path around which one has to evaluate the quantum fluctuations. A natural choice would be the classical path but it is much easier to take a straight line connecting the initial and final points

$$
x(t)=: x_{\text {straight }}(t)+\xi(t), \quad x_{\text {straight }}(t)=x_{a}+\frac{x_{b}-x_{a}}{t_{b}-t_{a}}\left(t-t_{a}\right) .
$$

Due to the boundary conditions $\xi\left(t_{a}\right)=\xi\left(t_{b}\right)=0$, the free action does not acquire a term linear in $\dot{\xi}(t)$ and one obtains

$$
\begin{aligned}
U\left(x_{b}, t_{b} ; x_{a}, t_{a}\right)= & {\left[\frac{m}{2 \pi i\left(t_{b}-t_{a}\right)}\right]^{d / 2} \exp \left[i \frac{m\left(x_{b}-x_{a}\right)^{2}}{2\left(t_{b}-t_{a}\right)}\right] } \\
& \times \mathcal{N}_{\xi} \int_{\xi\left(t_{a}\right)=0}^{\xi\left(t_{b}\right)=0} \mathcal{D}^{d} \xi(t) \exp \left\{i \int_{t_{a}}^{t_{b}} d t\left[\frac{m}{2} \dot{\xi}^{2}(t)-V\left(x_{\text {straight }}(t)+\xi(t), t\right)\right]\right\},
\end{aligned}
$$

where

$$
\mathcal{N}_{\xi}^{-1}=\int_{\xi\left(t_{a}\right)=0}^{\xi\left(t_{b}\right)=0} \mathcal{D}^{d} \xi(t) \exp \left\{i \int_{t_{a}}^{t_{b}} d t \frac{m}{2} \dot{\xi}^{2}(t)\right\}=\left[\frac{m}{2 \pi i\left(t_{b}-t_{a}\right)}\right]^{d / 2}
$$

Still the Feynman path integrals have to obey boundary conditions which sometimes make formal manipulations difficult. This is avoided if we switch to functional integration over velocities ${ }^{26}$ by 
multiplying Eq. (A3) with

$$
1=\prod_{k=1}^{N} \int d^{d} v_{k} \delta^{(d)}\left(\frac{x_{k}-x_{k-1}}{\epsilon}-v_{k}\right)=\epsilon^{d N} \prod_{k=1}^{N} \int d^{d} v_{k} \delta\left(x_{k}-x_{k-1}-\epsilon v_{k}\right) .
$$

As detailed in Ref. 2, one obtains

$$
\begin{aligned}
U\left(x_{b}, t_{b} ; x_{a}, t_{a}\right)= & \lim _{N \rightarrow \infty}\left(\frac{\epsilon m}{2 \pi i}\right)^{\frac{d N}{2}} \int d^{d} v_{1} \ldots d^{d} v_{N} \delta^{(d)}\left(x_{b}-x_{a}-\epsilon \sum_{k=1}^{N} v_{k}\right) \\
& \times \exp \left\{i \epsilon \sum_{k=1}^{N}\left[\frac{m}{2} v_{k}^{2}-V\left(x_{k}=x_{a}+\epsilon \sum_{j=1}^{k} v_{j}, t_{k}\right)\right]\right\} \\
\equiv & \mathcal{N}_{v} \int \mathcal{D}^{d} v(t) \delta^{(d)}\left(x_{b}-x_{a}-\int_{t_{a}}^{t_{b}} d t v(t)\right) \exp \left\{i \int_{t_{a}}^{t_{b}} d t\left[\frac{m}{2} v^{2}(t)-V(x(t), t)\right]\right\},
\end{aligned}
$$

where the position of the particle can be written symmetrically as

$$
x(t)=\frac{x_{a}+x_{b}}{2}+\frac{1}{2}\left[\int_{t_{a}}^{t} d \tau v(\tau)-\int_{t}^{t_{b}} d \tau v(\tau)\right]=: \frac{x_{a}+x_{b}}{2}+x_{v}(t) .
$$

The normalization of the velocity path integral is such that

$$
\mathcal{N}_{v}^{-1}=\int \mathcal{D}^{d} v(t) \exp \left\{i \int_{t_{a}}^{t_{b}} d t \frac{m}{2} v^{2}(t)\right\}=1
$$

and in its discretized form (A11) it consists of $N$ integrations instead of $(N-1)$ in the ordinary Feynman path integral. The quantum fluctuation around the mean position can also be expressed as

$$
x_{v}(t)=\frac{1}{2} \int_{t_{\min }}^{t_{\max }} d \tau \operatorname{sgn}(t-\tau) v(\tau), \quad t_{\min / \max }:=\min / \max \left(t_{a}, t_{b}\right), \quad t \in\left[t_{\min }, t_{\max }\right]
$$

where $\operatorname{sgn}(x)=2 \Theta(x)-1$ is the sign function. Note that $d x_{v}(t) / d t=v(t)$ since $d \operatorname{sgn}(t-\tau) / d t$ $=2 \delta(t-\tau)$.

Comparing Eqs. (A12) and (A4) one sees that a given velocity path integral over a general functional $F$ can always be written as an integral over an ordinary Feynman path integral

$$
\begin{aligned}
& \int \mathcal{D}^{d} v(t) \exp \left[i \int_{t_{a}}^{t_{b}} d t \frac{m}{2} v^{2}(t)\right] F\left[x_{v}(t)\right] \\
& =\int d^{d} z \int_{x\left(t_{a}\right)=-z / 2}^{x\left(t_{b}\right)=+z / 2} \mathcal{D}^{d} x(t) \exp \left[i \int_{t_{a}}^{t_{b}} d t \frac{m}{2} \dot{x}^{2}(t)\right] F[x(t)] .
\end{aligned}
$$

With a straight line reference path and the appropriate normalization factors (A14), (A5), and (A9) this becomes

$$
\begin{aligned}
\mathcal{N}_{v} \int \mathcal{D}^{d} v(t) & \exp \left[i \int_{t_{a}}^{t_{b}} d t \frac{m}{2} v^{2}(t)\right] F\left[x_{v}(t)\right]=\int d^{d} z\left[\frac{m}{2 \pi i\left(t_{b}-t_{a}\right)}\right]^{d / 2} \exp \left[i \frac{m}{2} \frac{z^{2}}{t_{b}-t_{a}}\right] \\
& \times \mathcal{N}_{\xi} \int_{\xi\left(t_{a}\right)=0}^{\xi\left(t_{b}\right)=0} \mathcal{D}^{d} \xi(t) \exp \left[i \int_{t_{a}}^{t_{b}} d t \frac{m}{2} \dot{\xi}^{2}(t)\right] F\left[-\frac{z}{2}+\frac{z}{t_{b}-t_{a}}\left(t-t_{a}\right)+\xi(t)\right] .
\end{aligned}
$$


While the composition law (A2) (or "semi-group" property) is built into the Lagrangian formulation and is easily proved in the discrete form by grouping the integrations into subproducts it is not immediately evident in the velocity path integral. Let us therefore evaluate

$$
\begin{aligned}
\left\langle x_{2}\left|\hat{U}\left(t_{2}, t_{1}\right) \hat{U}\left(t_{1}, t_{0}\right)\right| x_{0}\right\rangle=\int d^{d} x_{1}\left\langle x_{2}\left|\hat{U}\left(t_{2}, t_{1}\right)\right| x_{1}\right\rangle\left\langle x_{1}\left|\hat{U}\left(t_{1}, t_{0}\right)\right| x_{0}\right\rangle \\
=\int d^{d} x_{1} \prod_{k=1}^{2} \int \mathcal{D}^{d} v_{k} \delta^{(d)}\left(x_{k}-x_{k-1}-\int_{t_{k-1}}^{t_{k}} d \tau_{k} v_{k}\left(\tau_{k}\right)\right) \\
\quad \times \exp \left\{i \int_{t_{k-1}}^{t_{k}} d \tau_{k}\left[\frac{m}{2} v_{k}^{2}\left(\tau_{k}\right)-V\left(\frac{x_{k}+x_{k-1}}{2}+x_{v_{k}}\left(\tau_{k}\right), \tau_{k}\right)\right]\right\} .
\end{aligned}
$$

Performing the integration over $x_{1}$ with the help of the second $\delta$-function $(k=2$ in the product) gives

$$
\int \mathcal{D}^{d} v_{1} \mathcal{D}^{d} v_{2} \delta^{(d)}\left(y_{\delta}\right) \exp \left(i S_{0}\right) \exp \left\{-i \int_{t_{0}}^{t_{1}} d \tau_{1} V\left(y_{1}, \tau_{1}\right)-i \int_{t_{1}}^{t_{2}} d \tau_{2} V\left(y_{2}, \tau_{2}\right)\right\} \text {. }
$$

Here

$$
\begin{aligned}
& y_{\delta}=x_{2}-x_{0}-\int_{t_{1}}^{t_{0}} d \tau_{1} v_{1}\left(\tau_{1}\right)-\int_{t_{2}}^{t_{1}} d \tau_{2} v_{2}\left(\tau_{2}\right) \equiv x_{2}-x_{0}-\int_{t_{0}}^{t_{2}} d \tau v(\tau), \\
& S_{0}=\int_{t_{0}}^{t_{1}} d \tau_{1} \frac{m}{2} v_{1}^{2}\left(\tau_{1}\right)+\int_{t_{1}}^{t_{2}} d \tau_{2} \frac{m}{2} v_{2}^{2}\left(\tau_{2}\right) \equiv \int_{t_{0}}^{t_{2}} d \tau \frac{m}{2} v^{2}(\tau),
\end{aligned}
$$

if we define

$$
v(\tau):= \begin{cases}v_{1}(\tau) & \tau \in\left[t_{0}, t_{1}\right] \\ v_{2}(\tau) & \tau \in\left[t_{1}, t_{2}\right]\end{cases}
$$

The arguments of the potential terms are

$$
y_{1}\left(\tau_{1}\right)=\frac{x_{2}+x_{0}}{2}+x_{v_{1}}\left(\tau_{1}\right)-\frac{1}{2} \int_{t_{1}}^{t_{2}} d \tau v_{2}(\tau) \equiv \frac{x_{2}+x_{0}}{2}+x_{v}\left(\tau_{1}\right), \quad \tau_{1} \in\left[t_{0}, t_{1}\right]
$$

and, using the first $\delta$-function $(k=1)$ for determining $x_{1}$,

$$
y_{2}\left(\tau_{2}\right)=\frac{x_{2}+x_{0}}{2}+x_{v_{2}}\left(\tau_{2}\right)+\frac{1}{2} \int_{t_{0}}^{t_{1}} d \tau v_{1}(\tau) \equiv \frac{x_{2}+x_{0}}{2}+x_{v}\left(\tau_{2}\right), \quad \tau_{2} \in\left[t_{1}, t_{2}\right] .
$$

Thus,

$$
\begin{gathered}
\int d^{d} x_{1}\left\langle x_{2}\left|\hat{U}\left(t_{2}, t_{1}\right)\right| x_{1}\right\rangle\left\langle x_{1}\left|\hat{U}\left(t_{1}, t_{0}\right)\right| x_{0}\right\rangle= \\
\int \mathcal{D}^{d} v \delta^{(d)}\left(x_{2}-x_{0}-\int_{t_{0}}^{t_{2}} d \tau v(\tau)\right) \exp \left\{i \int_{t_{0}}^{t_{2}} d \tau\left[\frac{m}{2} v^{2}(\tau)-V\left(\frac{x_{2}+x_{0}}{2}+x_{v}(\tau), \tau\right)\right]\right\} \\
=\left\langle x_{2}\left|\hat{U}\left(t_{2}, t_{0}\right)\right| x_{0}\right\rangle
\end{gathered}
$$

proving the composition law. Unitarity of the time-evolution operator follows if the Hamiltonian is hermitean, or - in path-integral language - if the action is real:

$$
\left\langle x_{2}\left|\hat{U}\left(t_{2}, t_{1}\right) \hat{U}^{\dagger}\left(t_{0}=t_{2}, t_{1}\right)\right| x_{0}\right\rangle=\int d^{d} x_{1}\left\langle x_{2}\left|\hat{U}\left(t_{2}, t_{1}\right)\right| x_{1}\right\rangle\left\langle x_{0}\left|\hat{U}\left(t_{2}, t_{1}\right)\right| x_{1}\right\rangle^{\star}
$$


The last matrix element becomes

$$
\begin{aligned}
\left\langle x_{0}\left|\hat{U}\left(t_{2}, t_{1}\right)\right| x_{1}\right\rangle^{\star}= & \int \mathcal{D}^{d} v \delta^{(d)}\left(x_{0}-x_{1}-\int_{t_{1}}^{t_{2}} d \tau v(\tau)\right) \\
& \times \exp \left\{-i \int_{t_{1}}^{t_{2}} d \tau\left[\frac{m}{2} v^{2}(\tau)-V^{\star}\left(\frac{x_{1}+x_{0}}{2}+x_{v}(\tau), \tau\right)\right]\right\}
\end{aligned}
$$

and, if the potential is real $\left(V^{\star}=V\right)$, we have

$$
\begin{aligned}
\left\langle x_{0}\left|\hat{U}\left(t_{2}, t_{1}\right)\right| x_{1}\right\rangle^{\star} & =\int \mathcal{D}^{d} v \delta^{(d)}\left(x_{1}-x_{0}-\int_{t_{2}}^{t_{1}} d \tau v(\tau)\right) \exp \left[i \int_{t_{2}}^{t_{1}} d \tau \frac{m}{2} v^{2}(\tau)\right] \\
& \times \exp \left\{-i \int_{t_{2}}^{t_{1}} d \tau V\left(\frac{x_{1}+x_{0}}{2}+x_{v}(\tau), \tau\right)\right\} \equiv\left\langle x_{1}\left|\hat{U}\left(t_{1}, t_{2}\right)\right| x_{0}\right\rangle .
\end{aligned}
$$

Therefore,

$$
\begin{aligned}
\left\langle x_{2}\left|\hat{U}\left(t_{2}, t_{1}\right) \hat{U}^{\dagger}\left(t_{2}, t_{1}\right)\right| x_{0}\right\rangle & =\int d^{d} x_{1}\left\langle x_{2}\left|\hat{U}\left(t_{2}, t_{1}\right)\right| x_{1}\right\rangle\left\langle x_{1}\left|\hat{U}\left(t_{1}, t_{2}\right)\right| x_{0}\right\rangle \\
& =\left\langle x_{2}\left|\hat{U}\left(t_{2}, t_{2}\right)\right| x_{0}\right\rangle=\delta^{(d)}\left(x_{2}-x_{0}\right) \equiv\left\langle x_{2}|\hat{1}| x_{0}\right\rangle .
\end{aligned}
$$

\section{APPENDIX B: PROOF OF EQS. (14) AND (29)}

Here we recall the proof of these relations from standard (time-independent) scattering theory. Equation (14) is easily obtained by direct differentiation of Eq. (9) written with the explicit form of the outgoing scattering wave function $\left|\psi_{i}^{(+)}\right\rangle$

$$
T_{i \rightarrow f}=\left\langle\phi_{f}\left|\hat{V}_{\lambda}\left(1+\frac{1}{E-\hat{H}_{0}-\hat{V}_{\lambda}+i \epsilon}\right)\right| \phi_{i}\right\rangle
$$

and using

$$
\begin{aligned}
& \frac{\partial}{\partial \lambda} \frac{1}{E-\hat{H}_{0}-\hat{V}_{\lambda}+i \epsilon}=\frac{1}{E-\hat{H}_{0}-\hat{V}_{\lambda}+i \epsilon} \frac{\partial \hat{V}_{\lambda}}{\partial \lambda} \frac{1}{E-\hat{H}_{0}-\hat{V}_{\lambda}+i \epsilon} \\
& \left\langle\psi_{f}^{(-)}\right|=\left\langle\phi_{f}\right|\left(1+\hat{V}_{\lambda} \frac{1}{E-\hat{H}_{0}-\hat{V}_{\lambda}+i \epsilon}\right) .
\end{aligned}
$$

The energy derivative of the $T$-matrix in Eq. (29) is obtained by an energy-dependent scaling

$$
\hat{\mathbf{x}}=\lambda \mathbf{x}^{\prime}, \quad \hat{\mathbf{p}}=\frac{1}{\lambda} \hat{\mathbf{p}}, \quad \lambda=\frac{k_{0}}{k},
$$

where $k_{0}$ is a fixed wave number. Therefore, the plane waves become independent of the momentum $k$ and the Hamiltonian turns into

$$
\hat{H}=\frac{1}{\lambda^{2}}\left[\frac{\left(\hat{\mathbf{p}}^{\prime}\right)^{2}}{2 m}+\lambda^{2} V\left(\lambda \mathbf{x}^{\prime}\right)\right]=: \frac{1}{\lambda^{2}} \hat{H}^{\prime}
$$

Thus the system evolves with $\hat{H}^{\prime}$ and scaled times in time-dependent or with a scaled, fixed energy in time-independent scattering theory. Finally, from the volume element there is an additional factor 
$\lambda^{3}$ so that

$$
\frac{k}{k_{0}} T_{i \rightarrow f}=\int d^{3} x^{\prime} \phi_{f}^{\star}\left(\mathbf{x}^{\prime}\right) \lambda^{2} V\left(\lambda \mathbf{x}^{\prime}\right) \psi_{i}^{(+)}\left(\mathbf{x}^{\prime}\right)
$$

and all $k$-dependence resides now in the scaled potential $\lambda^{2} V\left(\lambda \mathbf{x}^{\prime}\right)$ for which we can apply Eq. (29). Performing the differentiation

$$
\frac{\partial}{\partial k}\left[\lambda^{2} V\left(\lambda \mathbf{x}^{\prime}\right)\right]=\frac{\partial \lambda}{\partial k}\left[2 \lambda V\left(\lambda \mathbf{x}^{\prime}\right)+\lambda^{2} \mathbf{x}^{\prime} \cdot \nabla V\left(\lambda \mathbf{x}^{\prime}\right)\right]=-\frac{\lambda^{3}}{k_{0}}\left[2 V\left(\lambda \mathbf{x}^{\prime}\right)+\lambda \mathbf{x}^{\prime} \cdot \nabla V\left(\lambda \mathbf{x}^{\prime}\right)\right]
$$

and transforming back to the unscaled coordinates gives Eq. (29). For a radially symmetric potential $V(r=|\mathbf{x}|)$ the (scattering) virial theorem depends on

$$
2 V(r)+r V^{\prime}(r)=\frac{1}{r} \frac{d}{d r}\left[r^{2} V(r)\right] .
$$

Some simple examples stand out: First, for a (repulsive) potential $V(r)=\alpha / r^{2}$ the rhs of Eq. (B8) vanishes and therefore its $T$-matrix (scattering amplitude) is proportional to $1 / k$ (which is correct as its phase shifts are energy-independent). Second, for a Coulomb potential $V(r)=\alpha / r$ one

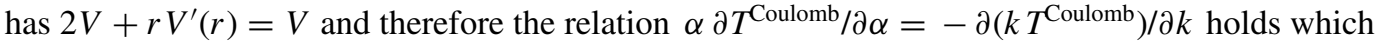
tells us that the Coulomb scattering amplitude is a function of the Sommerfeld parameter $\gamma=$ $m \alpha / k$ divided by $k$ (which is correct as can be seen from the exact expression, e.g., in Ref. 13, Eq. (B.25)).

${ }^{1}$ W. B. Campbell, P. Finkler, C. E. Jones, and M. N. Misheloff, "Path-integral formulation of scattering theory," Phys. Rev. D 12, 2363 (1975)

${ }^{2}$ R. Rosenfelder, "Path integrals for potential scattering," Phys. Rev. A 79, 012701 (2009); e-print arXiv:0806.3217 [nucl-th].

${ }^{3}$ J. Carron and R. Rosenfelder, "A new path-integral representation of the $T$-matrix in potential scattering," Phys. Lett. A 375, 3781 (2011); e-print arXiv:1107.3034 [nucl-th].

${ }^{4}$ G. V. Efimov, "Stationary Schrödinger equation in nonrelativistic quantum mechanics and the functional integral," Teor. Mat. Fiz. 171, 452 (2012) [Theor. Math. Phys. 171, 812 (2012)].

${ }^{5}$ G. V. Efimov, "Elastic scattering and path integral," e-print arXiv:1305.5658 [math-ph].

${ }^{6} \mathrm{~J}$. Carron, "Variational methods for path integral scattering," e-print arXiv:0903.0273v2 [nucl-th].

${ }^{7}$ J. Carron and R. Rosenfelder, "Variational approximations in a path-integral description of potential scattering," Eur. Phys. J. A 45, 193 (2010); e-print arXiv:0912.4429 [nucl-th].

${ }^{8} \mathrm{R}$. Rosenfelder, "Exact path-integral representations for the $T$-matrix in nonrelativistic potential scattering," Few-Body Syst. 49, 41 (2011); e-print arXiv:1008.1718 [nucl-th].

${ }^{9}$ L. S. Schulman, Techniques and Applications of Path Integration (John Wiley, New York, 1981).

${ }^{10}$ H. Kleinert, Path Integrals in Quantum Mechanics, Statistics, Polymer Physics and Financial Markets, 3rd ed. (World Scientific, Singapore, 2004).

${ }^{11}$ R. Rosenfelder, "Path Integrals in Quantum Physics/Pfadintegrale in der Quantenphysik," Lecture Notes (in German), e-print arXiv:1209.1315 [nucl-th].

${ }^{12}$ B. M. Barbashov, S. P. Kuleshov, V. A. Matveev, and A. N. Sisakian, "Eikonal approximation in quantum-field theory," Teor. Mat. Fiz. 3, 342 (1970) [Theor. Math. Phys. 3, 555 (1970)]; B. M. Barbashov, S. P. Kuleshov, V. A. Matveev, V. N. Pervushin, A. N. Sissakian, and A. N. Tavkhelidze, "Straight-line paths approximation for studying high-energy elastic and inelastic hadron collisions in quantum field theory," Phys. Lett. B 33, 484 (1970); V. A. Matveev and A. N. Tavkhelidze, "On the representation of scattering amplitudes as path integrals in quantum field theory," Teor. Mat. Fiz. 9, 44 (1971) [Theor. Math. Phys. 9, 968 (1971)].

${ }^{13}$ A. Messiah, Quantum Mechanics (North Holland, Amsterdam, 1965), Vol. II.

${ }^{14}$ L. S. Rodberg and R. M. Thaler, Introduction to the Quantum Theory of Scattering (Academic Press, New York, 1967).

${ }^{15}$ M. L. Goldberger and K. M. Watson, Collision Theory (John Wiley, New York, 1967).

${ }^{16}$ J. R. Taylor, Scattering Theory (John Wiley, New York, 1972).

${ }^{17}$ R. G. Newton, Scattering Theory of Waves and Particles, 2nd ed. (Springer, New York, Heidelberg, Berlin, 1985).

${ }^{18}$ In the following a quantum-mechanical operator is distinguished by a hat, 3-vectors are in bold face type, and a system of units is used where $\hbar=1$.

${ }^{19}$ Apart from an obvious minus sign missing in his Eq. (64) as can be seen by comparing Eqs. (60) and (63).

${ }^{20}$ Y. Tikochinsky, "Derivatives of phase shifts and binding energies by use of variational principles," J. Math. Phys. 11, 3019 (1970).

${ }^{21}$ The nomenclature indicates that $\rho$ is a function of $t$, but a functional of $\mathbf{v}$.

${ }^{22}$ Of course, this integration could be performed analytically on the rhs but for many applications the present form is preferable. 
${ }^{23}$ J. N. Demkov, Variational Principles in the Theory of Collisions (Pergamon Press, Oxford, 1963).

${ }^{24}$ F. Lenz, "Energiemomente von Streuphasen und Probleme der Streuung schneller Elektronen an Atomkernen," dissertation (in German), University of Freiburg (Germany), Fak. f. Phys. (1971).

${ }^{25}$ T. Adachi and T. Kotani, "An impact parameter representation of the scattering problem," Prog. Theor. Phys. 39, 430 (1968); 39, 785 (1968); M. M. Islam, "Impact parameter description of high energy scattering," in Boulder 1967, Lectures in Theoretical Physics, Vol. Xb, edited by A. O. Barut and W. E. Brittin (Interscience, New York, 1968), p. 97.

${ }^{26}$ D. M. Gitman and Sh. M. Shvartsman, "Path integrals over velocities in quantum mechanics," Phys. Lett. B 318, 122 (1993); e-print arXiv:hep-th/9310074; ibid. 331, 449 (1994) (erratum); W. da Cruz, J. Phys. A 30, 5225 (1997). 